\title{
Prolonged viral replication and longitudinal viral dynamic differences among respiratory syncytial virus infected infants
}

\author{
Monica E Brint ${ }^{1,4}$, Joshua M Hughes ${ }^{2,4}$, Aditya Shah ${ }^{3}$, Chelsea R Miller ${ }^{3}$, Lisa G Harrison ${ }^{1}$, Elizabeth A Meals ${ }^{1}$, \\ Jacqueline Blanch ${ }^{1}$, Charlotte R Thompson ${ }^{3}$, Stephania A Cormier ${ }^{1}$ and John P DeVincenzo ${ }^{3}$
}

BACKGROUND: Longitudinal respiratory syncytial virus (RSV) dynamics have not been well studied despite the existence of factors favoring prolonged RSV replication including high mutation rates allowing rapid evolution and potential escape from immune control. We therefore measured viral load in previously RSV-naive infants over prolonged time spans.

METHODS: During 2014-2015, quantitative nasal aspirates were collected from 51 RSV-PCR+ infants. Multiple parallel assessments of viral loads were quantified at each collected time point using a well-validated real-time quantitative reverse transcriptase polymerase chain reaction assay. After observing viral load rebound phenomenon in some infants, the viral dynamics of 27 infants with sufficient longitudinal viral load data points were analyzed using the pre-defined criteria for viral rebound. Additional analyses were performed comparing age with viral rebound, viral clearance rates, and viral load area-under-the-curve ( $\left.A \cup C_{V L}\right)$.

RESULTS: The 51 infants (303 nasal aspirate samples; mean of 5.9 per patient) exhibited slower than expected viral clearance. Lower age trended toward slower viral clearance and greater $A \cup C_{V L}$. Six infants had detectable viral loads $\geq 1$ month after symptom onset. Ten of twenty-seven evaluable subjects exhibited viral rebound and this rebound was age-dependent $(P=0.0259)$. All but one rebounder were $<70$ days old.

CONCLUSION: Infants struggle to control primary RSV infections allowing prolonged viral replication and previously undescribed viral rebound; likely representing viral mutational immune escape.

$\mathbf{R}$ espiratory syncytial virus (RSV) is the most common cause of lower respiratory tract infections in children and has no available vaccine or antiviral treatments (1). Although prematurity, immunodeficiency, chronic lung disease, and congenital heart disease are risk factors for severe RSV infections, the majority of children hospitalized for RSV are previously healthy, the major risk factor being young infant age (2). We have shown that higher mean viral loads correlate with greater disease severity and longer duration of hospitalization and symptoms (3-6). Young infants naturally infected with RSV have exhibited more prolonged viral shedding than their older counterparts (7). Properly performed viral culture can quantify replication-competent RSV, but it is a relatively insensitive test compared to PCR (8) and various factors elaborated within nasal secretions can neutralize culturable virus within those secretions $(1,9)$. Because RSV RNA is rapidly eliminated when RSV replication is effectively inhibited (10), a better tool to measure the actual viral replication appears to be quantifying RSV RNA species. We chose to quantify the RSV $N$ gene in this investigation. Furthermore, systematic longitudinal assessments of infant's viral loads have not previously been determined for longer than 3 days using real-time quantitative reverse transcriptase polymerase chain reaction (qRT-PCR) (11). In order to better understand the pathogenesis of RSV, we sought to describe the viral dynamics of RSV in infants over an extended time period. Therefore, from December 2014 to April 2015, the nasal aspirates of 51 naturally infected infants were quantitatively collected. The viral loads of these samples were measured with qRT-PCR during hospitalization and during multiple longitudinal follow-up outpatient visits for up to 1 month thereafter.

\section{METHODS}

\section{Subjects and Study Design}

Fifty-one naturally infected, hospitalized infants ( $<1$-year old) were enrolled after laboratory-confirmed RSV infection during the December 2014-April 2015 RSV season. In order to study viral clearance in previously healthy infants, subjects who had known bacteremia, immunodeficiency, hemodynamically significant congenital heart disease, previous oxygen use for $>7$ days, or had ever received corticosteroids, antiviral agents or palivizumab (Synagis, MedImmune, Gaithersburg, MD, USA) were excluded. The conduct of this research followed the University of Tennessee Health Science Center Institutional Review Board guidelines including appropriate informed consent.

\section{Sample Collection}

Nasal aspirate samples were collected daily during hospitalization and serially after discharge during study-specific visits on select days for up to 1 month after study enrollment. The scheduled study visits for sample collection include days $1,2,3,4,5,7,9,11,13,15,21$, and

\footnotetext{
${ }^{1}$ Department of Pediatrics, University of Tennessee Health Science Center, Memphis, Tennessee; ${ }^{2}$ College of Medicine, University of Tennessee Health Science Center, Memphis, Tennessee; ${ }^{3}$ Children's Foundation Research Institute, Memphis, Tennessee. Correspondence: John P. DeVincenzo (jdevincenzo@uthsc.edu)

${ }^{4}$ The first two authors contributed equally to this work.

Received 3 February 2017; accepted 21 June 2017; advance online publication 9 August 2017. doi:10.1038/pr.2017.173
} 
28. Study-specific visits were scheduled and accomplished regardless of the subject's clinical symptoms. If a subject missed their scheduled visit, arrangements were made to collect the sample within 1-2 days of their scheduled collection time. Samples were collected by trained, study-specific personnel using a standardized quantitative method as previously described (4). All subject information was removed; samples were assigned a designated lab number in order to blind study personnel during qRT-PCR assays.

Due to variable duration of hospitalization among subjects and loss to follow-up as outpatients, there was a lack of uniformity in the quantity of samples collected from different individuals. Parents of the subjects reported the onset of their child's symptoms during the initial study enrollment visit. The earliest onset of any associated symptom was used to normalize the timing of sample collection. The associated symptoms considered were fever, nasal congestion, coughing, and increased work of breathing.

\section{Viral RNA Extraction and Real-Time qRT-PCR}

A $200 \mu \mathrm{l}$ aliquot from the nasal aspirate sample was RNA extracted using an EZ1 Virus Minikit v2.0 and an automated EZ1 Advanced XL machine (Qiagen, Valencia, CA). Reverse transcription and a TaqMan-based real-time qRT-PCR assay was performed using the ABI Prism 7900HT and 7500Fast systems (Applied Biosystems International, Foster City, CA) (11). The qRT-PCR results were quantified using six duplicate-well, 10-fold dilution internal standard curves of identical micro-aliquots of quantitatively cultured RSV-A and RSV-B whole virus. The qRT-PCR results were reported as $\log _{10}$ plaque-forming unit equivalents per milliliter (Log PFUe/ml) (11). The qRT-PCR was performed in duplicate for each nasal aspirate sample, and separate runs were performed using custom primers specific for RSV-A and RSV-B subtypes (11). Additional second runs using this identical technique were performed. One of the RSV-Aspecific qRT-PCR runs failed, and limited sample volume prevented repeating the run. RSV-B-specific qRT-PCR results were reported as the geometric mean across all four individual wells from these two runs (Figure 1). The mean viral loads of the two runs were compared (Figure 2).

\section{Inter-Subject Consistency of Real-Time qRT-PCR Results}

Following reverse transcription, the nasal aspirate sample's cDNA was run in duplicate wells in the qRT-PCR assay (Figure 2). The detected viral loads from these duplicate wells were compared to determine the inter-sample reproducibility of the assay, showing minimal variability $\left(R^{2}=0.996\right.$, Figure 2a). For further determination of viral load measurement consistency, the mean viral loads from qRT-PCR runs 1 and 2 were compared. This analysis also showed a strong correlation between the two runs $\left(R^{2}=0.9234\right.$, Figure $2 \mathbf{b}$ ). This run-to-run analysis was only performed for the qRT-PCR assay using the RSV-B primers, because the second RSV-A run failed, leaving insufficient sample remaining.

\section{Analysis of Viral Load and Definition of Viral Load Rebound}

Enrolled subjects were evaluated for those having sufficient sample collections for longitudinal analysis as follows: nasal aspirate samples must have been collected for $\geq 5$ time points and $\geq 2$ of those time points must have occurred beyond the 8 th day after symptom onset. Twenty-seven subjects had sufficient longitudinal data. Before any data analysis was initiated, we defined criteria for viral load rebound as follows: a decrease in viral load over a course of $\geq 2$ time points before viral load nadir, the nadir must have occurred after day 8 of symptom onset, and an increase in viral load following the nadir must have occurred over $\geq 2$ time points, with 1 of those being $\geq 1$ Log PFUe/ml greater than the nadir.

\section{Analysis of Viral Load Clearance}

The viral load area-under-the-curve $\left(\mathrm{AUC}_{\mathrm{VL}}\right.$, Log PFUe $\times$ days $\left./ \mathrm{ml}\right)$ was calculated for the 27 longitudinally evaluable subjects. Viral load clearance within the first $48 \mathrm{~h}$ of study enrollment was determined by calculating the slope within that time frame. Of the 51 subjects, 40 subjects had sufficient samples for slope evaluation.

\section{Statistical Analysis}

Statistical analyses and figure construction were performed with GraphPad Prism Software v7.0 (La Jolla, CA). Variability between duplicate wells and separate qRT-PCR assay runs, as well as comparisons of age to AUC and viral clearance slopes were performed using linear regression. The subjects' ages (chronological and post conceptional) were normally distributed, and were compared to the presence of viral load rebound using unpaired $t$ tests. The AUC and viral clearance slopes were also compared to the presence of rebound using unpaired $t$-tests. Two-sided $\alpha$ of $<0.05$ were considered statistically significant.

\section{RESULTS}

\section{Characteristics of Study Population}

Subject characteristics were determined for both the entire data set of 51 subjects, as well as a subset of 27 subjects with sufficient data for individual longitudinal analysis (Table 1). The mean age of subjects at enrollment was $98.39 \pm 81.82$ (mean $\pm \mathrm{SD}$ ) days and $94.22 \pm 76.26$ days for the $n=51$ and $n=27$ data sets, respectively. On the basis of local RSV seasonality, ages, lack of previous respiratory hospitalization, and other subject history, all study subjects were enrolled during their first RSV season and therefore were likely experiencing their first RSV infection.

\section{Viral Persistence of Enrolled Subjects}

The 51 enrolled subjects were sampled for 301 separate times (mean 5.9 samples/subject), and the mean length of time over which samples were collected was 17.29 days after symptom onset. First sample collection occurred at a mean of 3 days after symptom onset. Ten of the subjects were only available for collection for $<7$ days. Of the infants with sufficient data collection at or after the representative time points, 39 of 41 (95\%) were still RSV+ at 1 week, 22 of 26 (85\%) at 2 weeks, and 6 of $16(38 \%)$ at 1 month after symptom onset (Figure 3a). The mean viral loads for each subject were individually analyzed for viral clearance ( $\Delta$ viral load/ $\Delta$ time) kinetics.

\section{Viral Load and Viral Dynamics}

Prior to data analysis and unblinding, 27 of 51 subjects were determined to have sufficient longitudinal points to analyze for viral rebound effect (five minimum time points with two or more time points occurring after day 8 of symptom onset). Ten of these twenty-seven subjects exhibited viral load rebound (defined in Methods section). An example of two individual patient's mean viral load curves, one exhibiting rebound and the other without rebound, is shown in Figure 3. Additional subjects' individual viral load curves lacking viral load rebound and exhibiting viral load rebound are shown in Figures 4 and 5, respectively.

After the assignment of viral rebound subject classification, the data were unblinded and the subjects' characteristics at enrollment were examined for association with viral load rebound beginning with the subjects' age at enrollment. The 


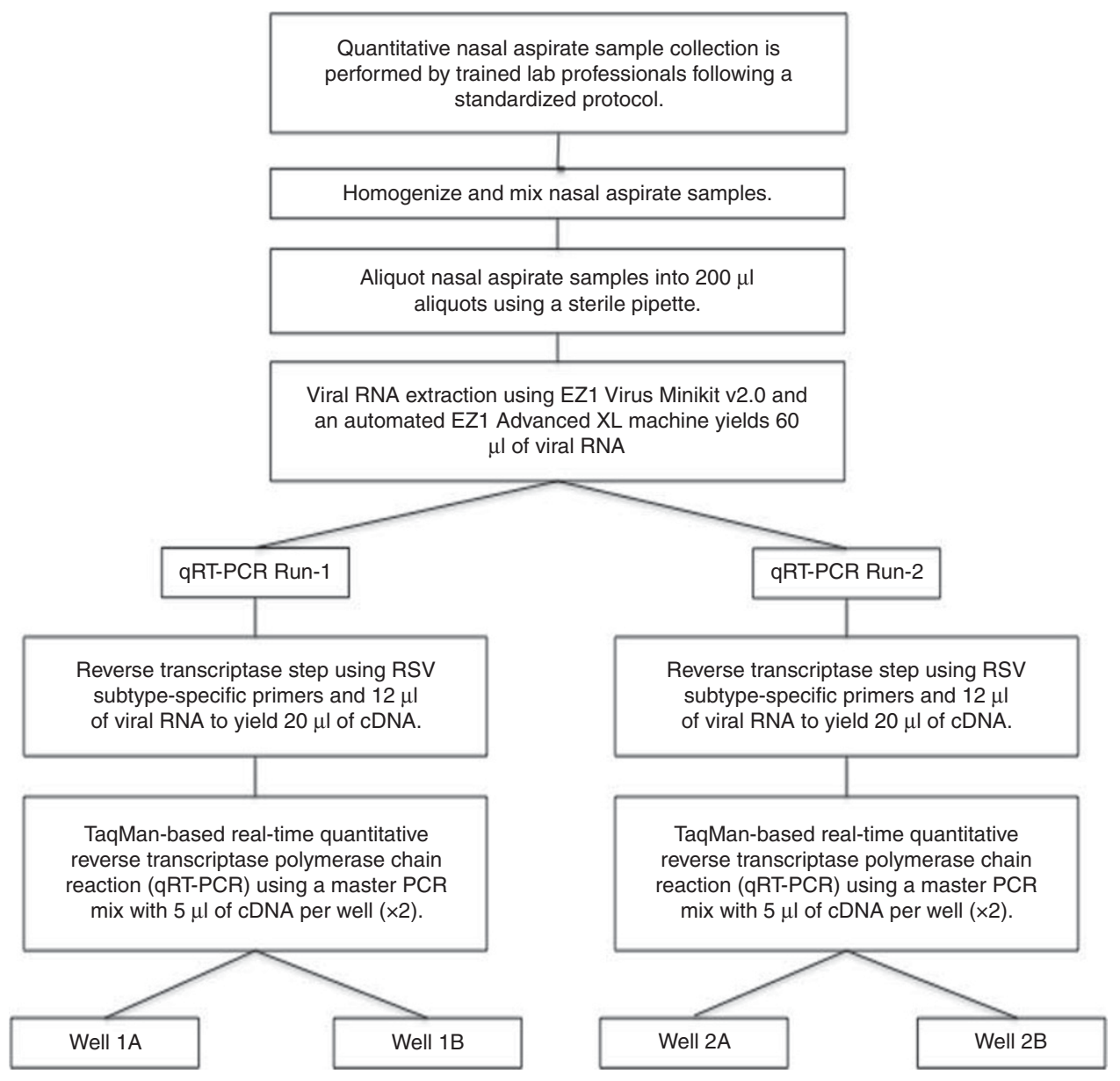

Figure 1. Study protocol for collecting and analyzing viral loads from nasal aspirate samples.

mean age of subjects with viral load rebound was significantly lower than the mean age of subjects with no viral load rebound (Figure 6; $P=0.0259$ ). All but one infant who had viral load rebound were $<70$ days of age (Figure 6). This significant association persisted when adjusting for postconceptional age (sum of gestational age and age at initial sample collection).

\section{Viral Clearance}

In order to investigate RSV clearance, the $\mathrm{AUC}_{\mathrm{VL}}$ of the 27 screened subjects was calculated. Potential correlations between these $\mathrm{AUC}_{\mathrm{VL}}$ values with age at enrollment, as well as with post-conceptional age were investigated. Lower age was associated with higher $\mathrm{AUC}_{\mathrm{VL}}$; however, this was not significant $(P=0.2536)$. There was a significantly higher mean AUC in the subjects who displayed viral rebound, compared to those who did not $(P=0.0035)$. Of these 27 subjects, 8 (30\%) had persistently detectable RSV at their last date of collection. Six of these eight subjects exhibited viral load rebound as previously described.

Viral clearance was also evaluated by analyzing the viral load slope within the first $48 \mathrm{~h}$ of study enrollment for the 40 subjects with evaluable time points. This analysis was performed in order to investigate a potential relationship between poor initial viral clearance and the presence of viral load rebound. Linear regression analysis was performed between viral clearance and age at enrollment, gestational age, as well as post-conceptional age as done with the $\mathrm{AUC}_{\mathrm{VL}}$ analysis. These analyses showed a trend toward more rapid viral clearance with increasing age at enrollment and postconceptional age; however, these findings were not significant $(P=0.4019$ and 0.6140 , respectively). Additionally, there was no significant association between the viral clearance slopes within the first $48 \mathrm{~h}$ of study enrollment and the presence of viral load rebound $(P=0.1395)$.

\section{DISCUSSION}

We report the first longitudinal sampling and systematic analysis of RSV load using modern molecular quantification techniques in large numbers of previously healthy infants. Several lines of evidence indicate real-time qRT-PCR reliably measures active RSV replication. The first evidence is that there is a very rapid lowering and disappearance of detectable qRT-PCR viral load in RSV-infected subjects after dosing with a potent RSV replication inhibitor (10). This shows RSV RNA does not persist in respiratory secretions after viral replication ceases. The second line of evidence is RSV mRNA can be quantitatively detected in human RSV infections throughout 

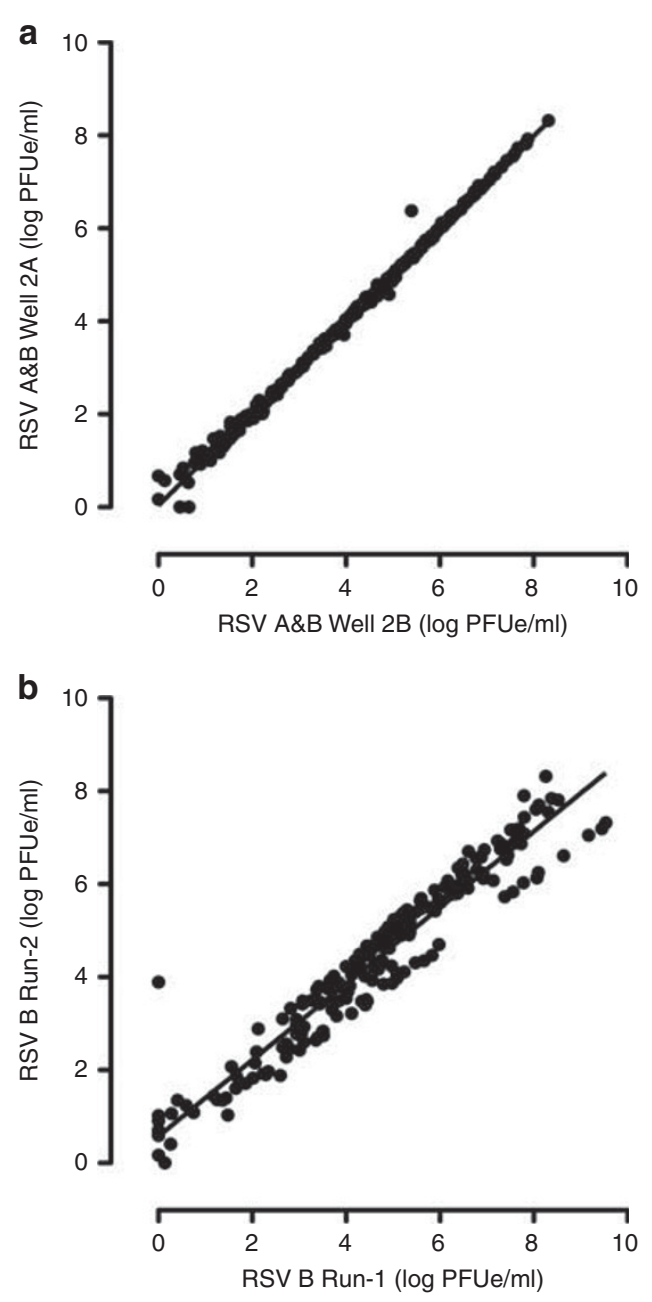

Figure 2. Evaluation of intrinsic variability in real-time quantitative reverse transcriptase polymerase chain reaction (qRT-PCR) assay. (a) The data points represent the mean viral load values (Log PFUe/ml) in duplicate wells during run-2 for RSV-A- and RSV-B-specific assays (Figure 1). Linear regression analysis showed that there was good correlation between the log values in the duplicate wells $\left(R^{2}=0.9959\right)$. (b) The data points represent the mean viral load values (Log PFUe/ml) of duplicate wells (mean of Wells $1 \mathrm{~A}$ and $1 \mathrm{~B}$, and mean of Wells $2 \mathrm{~A}$ and $2 B$ ) from the two runs of RSV-B-specific assays (Figure 1). There was good correlation between the mean well values between qRT-PCR run-1 and run-2 $\left(R^{2}=0.9234\right)$. No subject's qRT-PCR result on any sample switched from RSV-A to RSV-B nor from RSV-B to RSV-A.

the course of RSV qRT-PCR positivity even after culturenegativity occurs (9). The third line of evidence is that RSV culture from respiratory secretion of humans can become neutralized by the presence of antibodies within those secretions, showing RSV culture techniques measure falsely short durations of RSV replication $(9,12)$. Thus, RSV qRTPCR is an accurate measure of true RSV replication.

A single previous study described higher maximum viral loads occurring in hospitalized infants <1-month-old compared to those over the age of 1 month during 1974-1975 RSV season (7). This age-related finding suggests age may play a role in the initial viral load of naturally infected infants. One subject remained culture positive until their last collection time point at day 21 from their study enrollment, which may have suggested prolonged viral clearance in young infants. However, the study did not address the relative clearance kinetics of RSV in different age groups.

Despite some similar findings, this previous study differs from ours in methodology, study subject characteristics, and study design. Hall et al. (7) had a sample size of 23 subjects compared to our longitudinal study's sample size of 51 subjects. The previous study only followed up subjects in the outpatient setting if they were culture positive at hospital discharge. This may have biased the sample collection towards those who had a more severe infection and therefore required a longer duration of hospitalization. The previous study did not specify the number of subjects followed up in an outpatient setting until the subjects became culture negative. To contrast, our study collected an unbiased set of nasal aspirate samples during hospitalization and after their hospital discharge. The previous study did not collect nasal aspirate samples once the subject was RSV negative. Our study performed qRT-PCR blindly on all samples multiple times regardless of the predicted RSV result and all subjects were contacted for follow-up visits after hospital discharge according to our study visit schedule. Whereas the previous study collected only seven total samples after day 15 from enrollment, our longitudinal study collected 78 samples from 25 of 51 subjects at time points greater than day 15 from symptom onset. The previous study's methodology may have prevented Hall from evaluating clearance kinetics and detecting viral load rebound especially considering the threshold of detection of PCR is significantly better than $\mathrm{TCID}_{50}$ culture quantification.

The longitudinal viral dynamics of RSV have not previously been studied using qRT-PCR over time beyond the first few days $(6,11)$. In order to ensure longitudinal sample collections were not influenced by viral load results, qRT-PCR was performed on all samples $>6$ months after the 2014-2015 RSV season end. The subjects in this study were followed over a much more extensive time period and $30 \%$ of evaluable subjects showed detectable viral loads of $\geq 1 \mathrm{Log}$ PFUe/ml at 28-35 days after symptom onset. Overall, the 51 enrolled subjects exhibited very slow viral clearance kinetics. It was previously thought that RSV was rapidly cleared by the immune response within the first 1-2 weeks of symptom onset (13). The prolonged presence of replicating virus in our subjects suggests a delayed viral clearance in RSV-infected infants. This newly recognized prolonged viral replication corresponds well to previously recognized prolonged postacute symptoms and disease manifestations in RSV-infected infants, including prolonged cough and pulmonary function changes $(14,15)$.

We also observed that a frequent component of delayed viral clearance was viral load rebound occurring after the earliest estimated initiation of cell-mediated immune response, which generally first occurs around the 8th day of exposure (16). Further examination of individual subjects' viral load curves indicated that 10 of $27(37 \%)$ analyzed subjects expressed this rebound phenomenon. In order to 


\section{Articles | Brint et al.}

Table 1. Study subject demographics

\begin{tabular}{|c|c|c|c|c|}
\hline & \multicolumn{2}{|c|}{ Study subjects ${ }^{\mathrm{a}}(n=51)$} & \multicolumn{2}{|c|}{ Subjects for rebound analysis ${ }^{\mathrm{b}}(n=27)$} \\
\hline & \multicolumn{2}{|c|}{$n(\%)$} & \multicolumn{2}{|c|}{$n(\%)$} \\
\hline \multicolumn{5}{|l|}{ Race } \\
\hline Caucasian & \multicolumn{2}{|c|}{$13(25.49)$} & \multicolumn{2}{|c|}{$6(22.22)$} \\
\hline African-American & \multicolumn{2}{|c|}{$36(70.59)$} & \multicolumn{2}{|c|}{$19(70.37)$} \\
\hline Hispanic & \multicolumn{2}{|c|}{$1(1.96)$} & \multicolumn{2}{|c|}{$1(3.70)$} \\
\hline Other & \multicolumn{2}{|c|}{$1(1.96)$} & \multicolumn{2}{|c|}{$1(3.70)$} \\
\hline \multicolumn{5}{|l|}{ Sex } \\
\hline Male & \multicolumn{2}{|c|}{$32(62.75)$} & \multicolumn{2}{|c|}{$17(62.96)$} \\
\hline Female & \multicolumn{2}{|c|}{$19(37.25)$} & \multicolumn{2}{|c|}{$10(37.04)$} \\
\hline Subjects requiring oxygen supplementation & \multicolumn{2}{|c|}{$36(70.59)$} & \multicolumn{2}{|c|}{$16(59.26)$} \\
\hline Subjects In ICU & \multicolumn{2}{|c|}{$5(9.80)$} & \multicolumn{2}{|c|}{$4(14.81)$} \\
\hline Subjects requiring mechanical ventilation & \multicolumn{2}{|c|}{$3(5.88)$} & \multicolumn{2}{|c|}{$3(11.11)$} \\
\hline Subjects with family history of asthma & \multicolumn{2}{|c|}{$30(58.82)$} & \multicolumn{2}{|c|}{$18(66.67)$} \\
\hline \multirow[t]{3}{*}{ Subjects with family history of eczema } & \multicolumn{2}{|c|}{$16(31.37)$} & \multicolumn{2}{|c|}{$8(29.63)$} \\
\hline & \multicolumn{4}{|c|}{ Study subject clinical characteristics } \\
\hline & Mean $( \pm S D)$ & $\mathrm{IQR}$ & Mean $( \pm S D)$ & IQR \\
\hline Age at admission (days) & $98.39( \pm 81.82)$ & 87 & $94.22( \pm 76.26)$ & 116 \\
\hline Age at NW collection (days) & $99.45( \pm 81.80)$ & 86 & $95.30( \pm 76.18)$ & 116 \\
\hline Gestational age (weeks) & $37.53( \pm 2.33)$ & 3 & $37.76( \pm 2.46)$ & 4 \\
\hline Birth weight (g) & $2873( \pm 725.3)$ & 899 & $2863( \pm 778.3)$ & 850 \\
\hline Hospital length of stay (days) & $5.92( \pm 3.24)$ & 3 & $6.70( \pm 4.08)$ & 5 \\
\hline Duration of symptom onset (days) & $4.14( \pm 2.00)$ & 2 & $4.22( \pm 2.28)$ & 2 \\
\hline Number of children in household & $2.12( \pm 1.63)$ & 3 & $2.07( \pm 1.66)$ & 2 \\
\hline Number of household members & $5.08( \pm 1.72)$ & 3 & $4.89( \pm 1.65)$ & 2 \\
\hline
\end{tabular}

ICU, intensive care unit; IQR, interquartile range; NW, nasal wash; SD, standard deviations.

asubjects who had known bacteremia, immunodeficiency, hemodynamically significant congenital heart disease, previous oxygen use for $>7$ days, or had ever received corticosteroids, antiviral agents, or palivizumab (Synagis) were excluded.

${ }^{b}$ Represents those subjects that were preselected to have sufficient viral load data for longitudinal evaluation (see Methods).

reduce the probability of false classification of viral rebound brought about by sampling or procedural variability, an objective biologically based conservative definition of viral load rebound was applied blindly to all 27 subjects who had repeated measurements of viral load from quantitatively collected samples. On the basis of the well-established association of young infant age and severe RSV disease $(4,17)$, age at enrollment was evaluated with respect to those showing viral load rebound (Figure 6). Because RSV infection is so common during infancy, and because RSV exposure occurs at different ages during infancy, this natural viral infection can be uniquely used to interrogate the ontogeny of the infant immune response. The significantly lower mean age of subjects displaying viral load rebound suggests an agerelated functional maturation of infant cell-mediated antiviral immune responses.

The possibility that a re-infection produced our observed rebound phenomenon is extremely low. Within our individual subjects' longitudinal sample collections, we observed no switching of RSV subtype, based on subtype-specific probes to
$\mathrm{N}$-gene region, despite the high frequency of RSV-A and RSV-B co-circulating within the 2014-2015 season's epidemic (Figure 2). In future studies, the potential presence of RSV genotype switching within individual subjects might be further explored by performing whole-genome deep sequencing on subjects' longitudinal samples.

Viral clearance within the first $48 \mathrm{~h}$ was examined for significant differences in initial antiviral immune responses. There were no statistically significant correlations between initial viral clearance and age or the presence of viral load rebound. However, using the same assay, the viral load AUC in naturally infected infants is statistically higher than in adults who are experimentally infected with RSV $(P<0.0001)$ (5). Due to the complications of the viral load rebound phenomena and eight subjects having detectable viral loads at the last collection time point, the utility of evaluating viral load AUC vs. host differences was difficult.

Our study had certain limitations. For instance, the subject population for this study encompasses infants hospitalized with RSV, but does not include infants encountering RSV in 

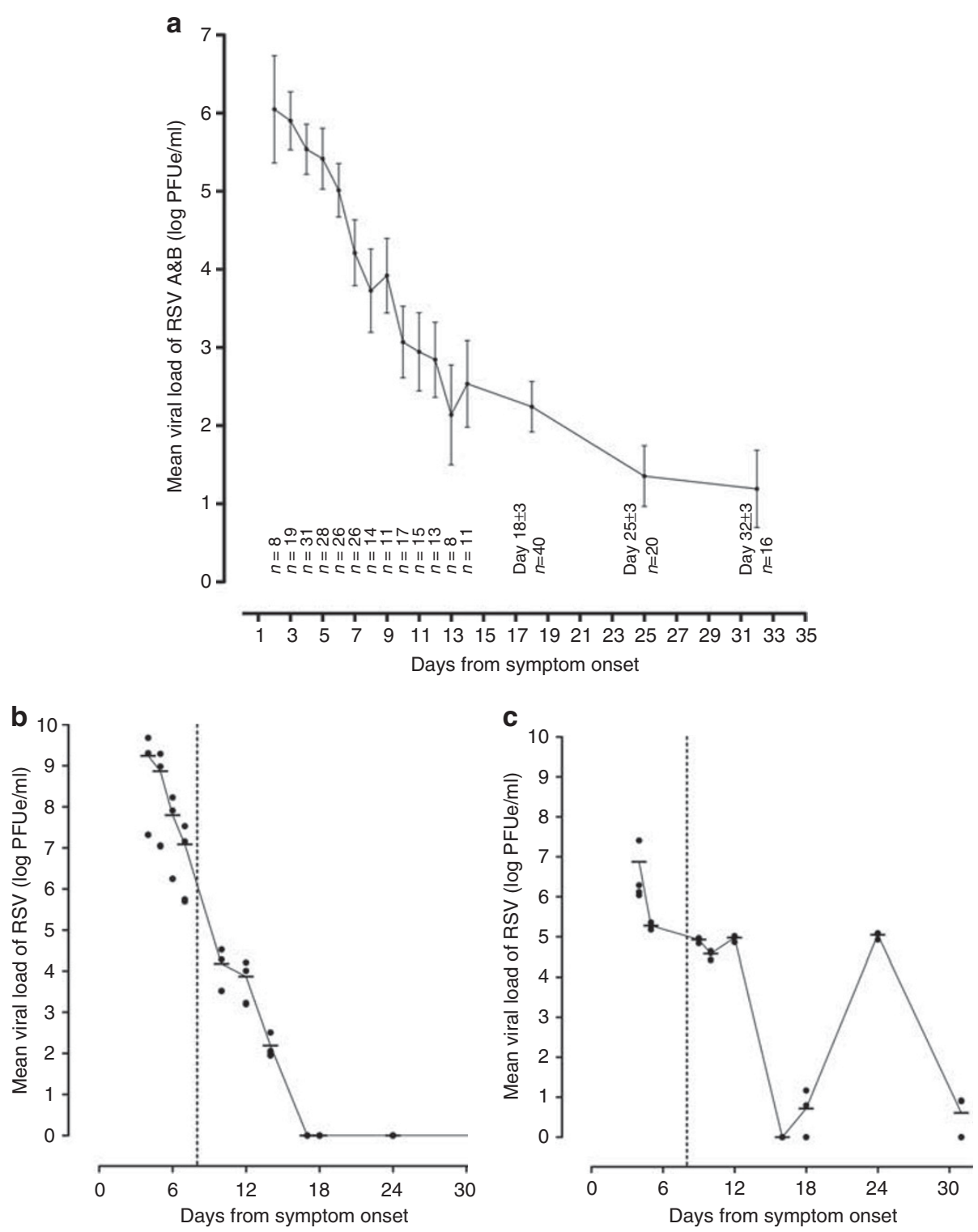

Figure 3. Prolonged viral replication and viral load rebound. (a) Mean viral load of RSV-A and $-B( \pm$ SEM) measured in log plaque-forming unit equivalents per milliliter (Log PFUe/ml). Each data point represents the geometric mean viral load of nasal aspirate samples for a specific day from symptom onset. The corresponding sample size is represented below each data point. (b) A representative individual subject viral load graph showing no viral load rebound. (c) A representative individual subject viral load graph showing viral load rebound. The vertical dashed line on day 8 from symptom onset represents the estimated onset of the subject's first cell-mediated immune response. The data points (filled circles) represent the viral load for individual wells, and horizontal dashes represent the geometric mean viral load for a specific day from symptom onset.

outpatient and home environments. Although the disease severity is clearly higher in hospitalized subjects, the study of this subpopulation has clear clinical relevance. During the course of study, frequent nasal aspirate samples were collected, but due to a lack of existing validated symptom-scoring instruments for outpatients the corresponding symptoms were not able to be documented. In future studies, symptom scores will be implemented alongside the sample collection to address this issue. Additionally, there are logistical difficulties in collecting symptom information because the subjects cannot self-report their symptoms and there is not a validated method for parental reporting of their child's symptoms. We have recently shown through within-host longitudinal sampling and whole-viral genome deep sequencing that RSV genome diversity evolves directionally within a single RSV infection in response to the institution of an infant immune response (18). This diversity includes amino-acid sites within the second hypervariable region of RSV-G, which is used for viral genotype assignment. In future studies of this clinical cohort, we plan to utilize whole-genome viral longitudinal within-host deep sequencing to evaluate the mechanisms of the viral load rebound phenomenon, examining whether viral diversity 

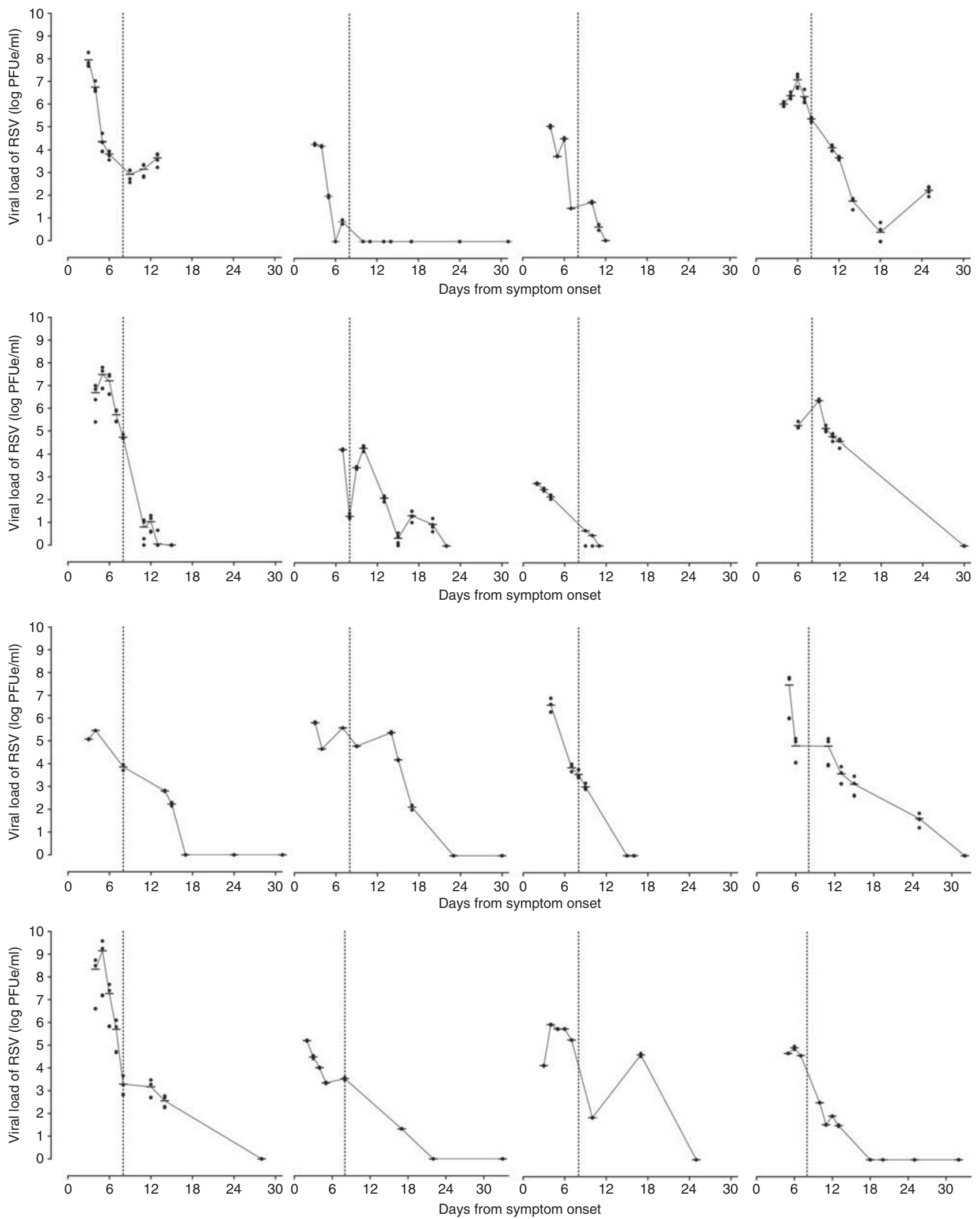

Figure 4. Individual subject viral load graphs showing lack of viral load rebound. Included in the figure are all subjects with sufficient data points for longitudinal analysis. Subjects were excluded if they had fewer than five data points or had fewer than two data points occurring after day 8 from symptom onset. The vertical dashed line on day 8 from symptom onset represents the estimated onset of the subject's first cell-mediated immune response. The data points (filled circles) represent the viral load for individual wells, and the horizontal dashes represent the geometric mean viral load for a specific day from symptom onset. 

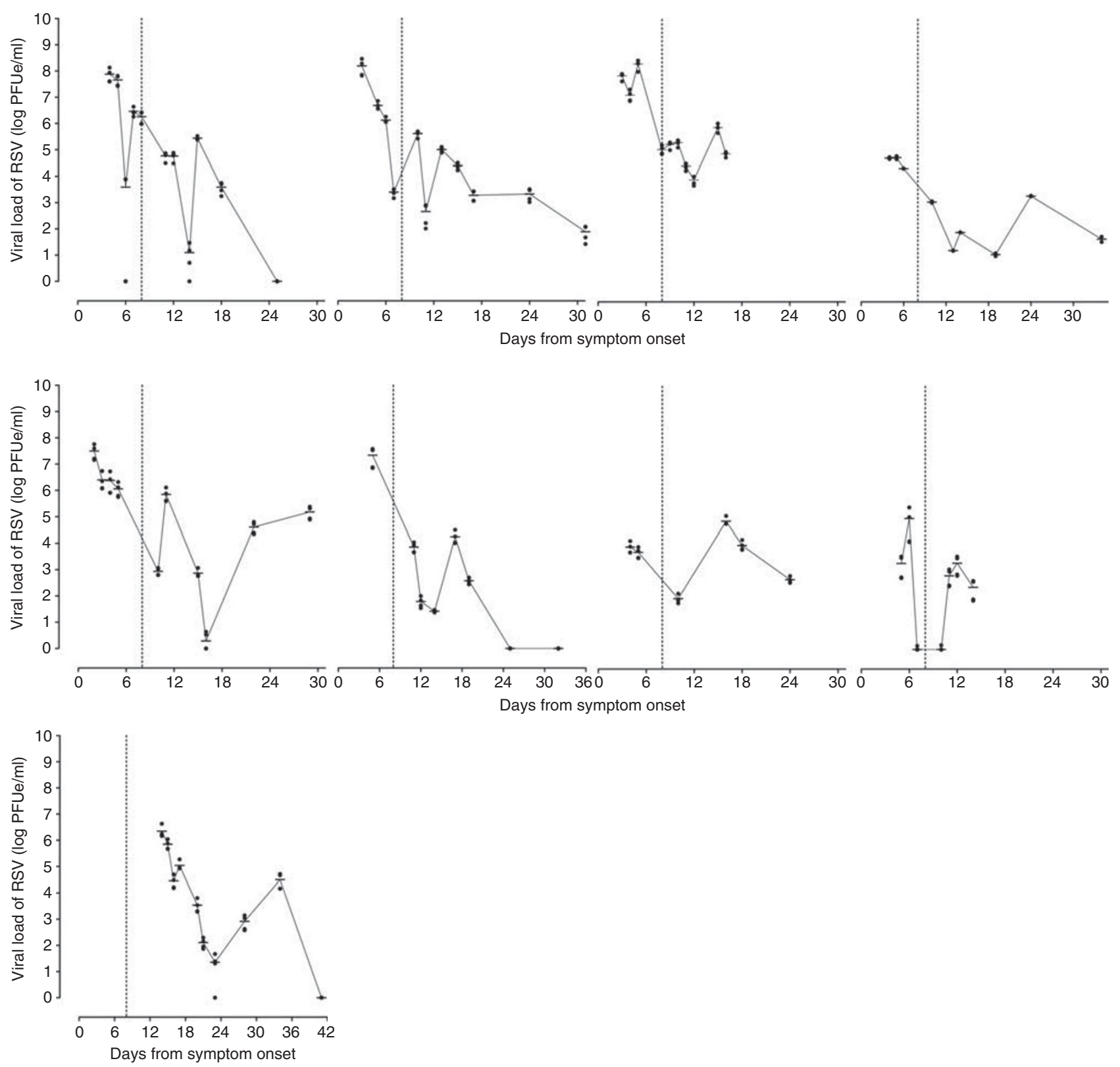

Figure 5. Individual subject viral load graphs showing viral load rebound. Included in the figure are all subjects with sufficient data points for longitudinal analysis. Subjects were excluded if they had fewer than five data points or had fewer than two data points occurring after day 8 from symptom onset. The vertical dashed line on day 8 from symptom onset represents the estimated onset of the subject's first cell-mediated immune response. The data points (filled circles) represent the viral load for individual wells, and the horizontal dashes represent the geometric mean viral load for a specific day from symptom onset.

during rebound occurs at recognized T- and B-cell epitope sites (19). Additionally, the future deep genome sequence analysis of the second hypervariable region of $G$ protein in our study samples may allow us to distinguish between immune escape mutations in the original RSV infection and the presence of a second RSV infection.

Past studies have shown that there is a link between severe RSV infections in early life and the development of chronic wheezing and asthma (20). Future investigations of the relationship between prolonged viral load presence and chronic wheezing would provide further insight into the clinical relevance of prolonged viral replication and viral load rebound. This analysis may also be paired with the evaluation of immune response differences in infants with delayed viral clearance.

In conclusion, RSV replicates more extensively than previously recognized in infants and much longer than adults. Viral load rebound is frequent among subjects and especially in those displaying delayed viral load clearance. This phenomenon of viral load rebound was not previously 


\section{Articles | Brint et al.}

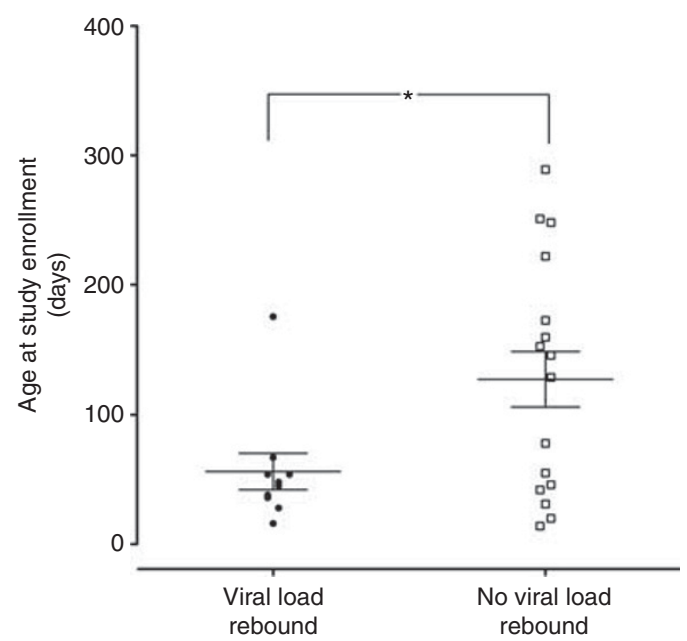

Figure 6. Age at study enrollment and viral load rebound. Mean age $( \pm$ SD) at study enrollment for 27 screened subjects (subjects with viral load rebound, $56.2 \pm 14.07$ days $(n=10)$; subjects with no viral load rebound, $127.5 \pm 21.4$ days $(n=17) ; P=0.0259)$. Each data symbol represents an individual subject's age at study enrollment. Following evaluation for Gaussian distribution using a D'Agostino Normality test, an unpaired $t$-test (two-tailed) was performed to compare the mean ages between the subjects with viral load rebound and the subjects with no viral load rebound; ${ }^{*} P=0.0259$.

described in any acute viral illness and appears to be occurring after the initiation of cell-mediated immune responses, in an age-dependent manner. Establishing the mechanisms and clinical relevance of prolonged viral replication and viral load rebound will help us to understand RSV disease pathogenesis. Our findings have clear relevance to the development of antiviral therapies, potentially strengthening their utility in a clinical setting.

\section{ACKNOWLEDGMENTS}

We thank the parents and caregivers of the participating infants for their altruism. Without whom, we could not make advancements toward a better understanding of RSV infections, their treatment, and prevention. Dr. DeVincenzo and Dr. Cormier also thank the Department of Pediatrics at University of Tennessee Health Science Center, the Department of Microbiology, Immunology, and Biochemistry at University of Tennessee Health Science Center, Children's Foundation Research Institute and Le Bonheur Children's Hospital for the opportunity to conduct our research and expand the knowledge of RSV infections in the pediatric population.

\section{STATEMENT OF FINANCIAL SUPPORT}

This work was supported by internal funds (Grant number: RO73230070) from the DeVincenzo laboratory and University of Tennessee Health Science Center; no external financial support was received for this study. J. $\mathrm{H}$. was supported by a University of Tennessee Health Science Center College of Medicine-sponsored Medical Students Research Fellowship funded by the University of Tennessee Department of Pediatrics and the LeBonheur Children's Hospital.

Disclosure: The authors declare no conflict of interest.

\section{REFERENCES}

1. Simões EA, Devincenzo JP, Boeckh M, et al. Challenges and opportunities in developing respiratory syncytial virus therapeutics. J Infect Dis 2015;211(Suppl 1):S1-20.

2. Boyce TG, Mellen BG, Mitchel EF Jr, Wright PF, Griffin MR. Rates of hospitalization for respiratory syncytial virus infection among children in medicaid. J Pediatr 2000;137:865-70.

3. Buckingham SC, Bush AJ, Devincenzo JP. Nasal quantity of respiratory syncytical virus correlates with disease severity in hospitalized infants. Pediatr Infect Dis J 2000;19:113-7.

4. Devincenzo JP, El Saleeby CM, Bush AJ. Respiratory syncytial virus load predicts disease severity in previously healthy infants. J Infect Dis 2005;191:1861-8.

5. Devincenzo JP, Wilkinson T, Vaishnaw A, et al. Viral load drives disease in humans experimentally infected with respiratory syncytial virus. Am J Respir Crit Care Med 2010;182:1305-14.

6. El Saleeby CM, Bush AJ, Harrison LM, Aitken JA, Devincenzo JP. Respiratory syncytial virus load, viral dynamics, and disease severity in previously healthy naturally infected children. J Infect Dis 2011;204: 996-1002.

7. Hall CB, Douglas RG, Geiman JM. Respiratory syncytial virus infections in infants: quantitation and duration of shedding. J Pediatr 1976;89:11-5.

8. Ginocchio CC. Strengths and weaknesses of FDA-approved/cleared diagnostic devices for the molecular detection of respiratory pathogens. Clin Infect Dis 2011;52 (Suppl 4): S312-25.

9. Bagga B, Cehelsky JE, Vaishnaw A, et al. Effect of preexisting serum and mucosal antibody on experimental respiratory syncytial virus (RSV) challenge and infection of adults. J Infect Dis 2015;212:1719-25.

10. Devincenzo JP, Mcclure MW, Symons JA, et al. Activity of oral ALS-008176 in a Respiratory Syncytial Virus Challenge Study. N Engl J Med 2015;373:2048-58.

11. Perkins SM, Webb DL, Torrance SA, et al. Comparison of a real-time reverse transcriptase PCR assay and a culture technique for quantitative assessment of viral load in children naturally infected with respiratory syncytial virus. J Clin Microbiol 2005;43:2356-62.

12. Lagos R, Devincenzo JP, Muñoz A, et al. Safety and antiviral activity of motavizumab, a respiratory syncytial virus (RSV)-specific humanized monoclonal antibody, when administered to RSV-infected children. Pediatr Infect Dis J 2009;28:835-7.

13. Collins PL, Graham BS. Viral and host factors in human respiratory syncytial virus pathogenesis. J Virol 2008;82:2040-55.

14. Bisgaard H, Flores-Nunez A, Goh A, et al. Study of montelukast for the treatment of respiratory symptoms of post-respiratory syncytial virus bronchiolitis in children. Am J Respir Crit Care Med 2008;178:854-60.

15. Eiland LS. Respiratory syncytial virus: diagnosis, treatment and prevention. J Pediatr Pharmacol Ther 2009;14:75-85.

16. Thomas PG, Keating R, Hulse-Post DJ, Doherty PC. Cell-mediated protection in influenza infection. Emerg Infect Dis 2006;12:48-54.

17. Pickles RJ, Devincenzo JP. Respiratory syncytial virus (RSV) and its propensity for causing bronchiolitis. J Pathol 2015;235:266-76.

18. Grad YH, Newman R, Zody M, et al. Within-host whole-genome deep sequencing and diversity analysis of human respiratory syncytial virus infection reveals dynamics of genomic diversity in the absence and presence of immune pressure. J Virol 2014;88:7286-93.

19. Kim-Hoehamer Y-I, Lau J, Murphy R, et al. Deep-sequencing of longitudinal samples from RSV-infected adults and infants reveals heterogeneous dynamics of within-host viral diversification. J Immunol 2016;196(Suppl 1):80.8

20. Feldman AS, He Y, Moore ML, Hershenson MB, Hartert TV. Toward primary prevention of asthma. Reviewing the evidence for early-life respiratory viral infections as modifiable risk factors to prevent childhood asthma. Am J Respir Crit Care Med 2015;191:34-44. 